\title{
Habitat and landscape factors influence pollinators in a tropical megacity, Bangkok, Thailand
}

\author{
Alyssa B. Stewart ${ }^{\text {Corresp., }}{ }^{1}$, Tuanjit Sritongchuay ${ }^{2}{ }^{\text {, Piyakarn Teartisup }}{ }^{3}$, Sakonwan Kaewsomboon ${ }^{1}$, Sara \\ Bumrungsri ${ }^{4}$ \\ 1 Department of Plant Science, Faculty of Science, Mahidol University, Bangkok, Thailand \\ 2 Center for Integrative Conservation, Xishuangbanna Tropical Botanical Garden, Chinese Academy of Sciences, Meglun, Mengla, Yunnan, China \\ 3 Faculty of Environment and Resource Studies, Mahidol University, Salaya, Nakhon Pathom, Thailand \\ 4 Department of Biology, Faculty of Science, Prince of Songkla University, Hat Yai, Songkhla, Thailand \\ Corresponding Author: Alyssa B. Stewart \\ Email address: 4lyssa@gmail.com
}

Background. Pollinators are well known for the ecosystem services they provide, and while urban areas are generally perceived as low-quality habitat for most wildlife, these cities often support a surprising degree of pollinator diversity. The current rapid growth of urban areas and concern over global pollinator declines have spurred numerous studies examining pollinator communities in temperate cities, but knowledge about tropical urban pollinators remains scarce.

Methods. This study investigated the effects of habitat and landscape factors on pollinator richness and abundance in a highly-populated, tropical city: Bangkok, Thailand. We conducted pollinator observations in 52 green areas throughout the city and collected data on patch size, floral abundance, plant richness, location type, and percent vegetation at 5 spatial scales.

Results. Of the 18,793 pollinators observed, over $98 \%$ were bees. Both patch size and floral abundance generally had positive effects on pollinators, although there was a significant interaction between the two factors; these findings were generally consistent across all focal taxa (Tetragonula stingless bees, Apis honey bees, Xylocopa carpenter bees, and butterflies).

Discussion. Our results demonstrate the importance of maintaining large green areas in cities, since small green areas supported few pollinators, even when floral resources were abundant. Moreover, most pollinator taxa utilized a variety of location types (e.g., public parks, school campuses, temple grounds), with the exception of butterflies, which preferred parks. Our findings are generally consistent with those of temperate urban studies, but additional studies in the tropics are needed before global patterns can be assessed. 


\section{Habitat and landscape factors influence pollinators in a tropical}

\section{2 megacity, Bangkok, Thailand}

3 Alyssa B. Stewart ${ }^{1}$, Tuanjit Sritongchuay ${ }^{2}$, Piyakarn Teartisup ${ }^{3}$, Sakonwan Kaewsomboon ${ }^{1}$, and

4 Sara Bumrungsri ${ }^{4}$

5

$6 \quad{ }^{1}$ Department of Plant Science, Faculty of Science, Mahidol University, Bangkok 10400,

7 Thailand

$8 \quad{ }^{2}$ Center for Integrative Conservation, Xishuangbanna Tropical Botanical Garden, Chinese

9 Academy of Sciences, Meglun, Mengla, Yunnan 666303, China

$10{ }^{3}$ Faculty of Environment and Resource Studies, Mahidol University, Salaya, Nakhon Pathom

1173170 , Thailand

$12{ }^{4}$ Department of Biology, Faculty of Science, Prince of Songkla University, Hat Yai, Songkhla

13 90112, Thailand

15 Corresponding author:

16 Alyssa Stewart

17 Email address: alyssa.ste@mahidol.edu 


\section{ABSTRACT}

Background. Pollinators are well known for the ecosystem services they provide, and while urban areas are generally perceived as low-quality habitat for most wildlife, these cities often support a surprising degree of pollinator diversity. The current rapid growth of urban areas and concern over global pollinator declines have spurred numerous studies examining pollinator communities in temperate cities, but knowledge about tropical urban pollinators remains scarce.

Methods. This study investigated the effects of habitat and landscape factors on pollinator richness and abundance in a highly-populated, tropical city: Bangkok, Thailand. We conducted pollinator observations in 52 green areas throughout the city and collected data on patch size, floral abundance, plant richness, location type, and percent vegetation at 5 spatial scales. Results. Of the 18,793 pollinators observed, over 98\% were bees. Both patch size and floral abundance generally had positive effects on pollinators, although there was a significant interaction between the two factors; these findings were generally consistent across all focal taxa (Tetragonula stingless bees, Apis honey bees, Xylocopa carpenter bees, and butterflies).

Discussion. Our results demonstrate the importance of maintaining large green areas in cities, since small green areas supported few pollinators, even when floral resources were abundant. Moreover, most pollinator taxa utilized a variety of location types (e.g., public parks, school campuses, temple grounds), with the exception of butterflies, which preferred parks. Our findings are generally consistent with those of temperate urban studies, but additional studies in the tropics are needed before global patterns can be assessed. 
42

43

44

45

46

47

48

\section{INTRODUCTION}

3

4

45

concentrated in cities (Grimm et al. 2008; Seto et al. 2012). Such growth has sparked a greater interest in urban ecology; a Web of Science search (conducted May 2018) for the terms "urban*" and "ecolog*" revealed 1,915 results from 2003-2007, 4,011 results from 2008-2012, and 8,305 results from 2013-2017. Thus, the literature in this field has doubled approximately every five years. This growing interest has stemmed, in part, as ecologists have come to recognize, (1) the large impacts of urban areas on local biodiversity, (2) the importance of preserving biodiversity in cities, and (3) the value of cities in modelling future biodiversity patterns, as urban areas are predicted to continue expanding worldwide (Grimm et al. 2008; Bates et al. 2011; Robinson \& Lundholm 2012; Seto et al. 2012).

One area of urban ecology that has received considerable attention is that of pollinator communities in cities. This interest is driven by a combination of general appreciation for the importance of pollinators (Klein et al. 2007; Ollerton et al. 2011), and growing concern over global pollinator declines (Potts et al. 2010 and references therein). Urbanization is generally predicted to have negative effects on pollinators (Cariveau \& Winfree 2015), with studies demonstrating greater habitat isolation in cities (Ferreira et al. 2013), reduced pollinator movement (Bhattacharya et al. 2013), and consequent reproductive isolation and higher rates of selfing in plants (Harrison \& Winfree 2015). Yet cities are often centers of high plant diversity and floral abundance (due to the cultivation of many exotic flowering plant species), which can 
63 lead to plentiful food and nesting resources (McFrederick \& LeBuhn 2006) and sometimes a

64 surprising degree of pollinator diversity (Hennig \& Ghazoul 2011; Hall et al. 2016).

65 influence pollinator communities. Some variables appear to be consistently important across diverse countries and pollinator taxa, such as floral abundance (Ahrné et al. 2009; Bates et al. 2011; Hennig \& Ghazoul 2011) and plant diversity (Kearns \& Oliveras 2009; Bates et al. 2011; Hennig \& Ghazoul 2011; Hülsmann et al. 2015). Other variables have received mixed support (such as patch size - McFrederick \& LeBuhn 2006; Ahrné et al. 2009; Hennig \& Ghazoul 2011;

Pardee \& Philpott 2014), or appear to be important for only specific pollinator taxa (such as the amount of woody vegetation - Pardee \& Philpott 2014). Overall, the results seem to indicate there is some variation across locations and pollinator taxa, yet several patterns appear to be fairly universal.

However, before we can assess global patterns of pollinator responses to urbanization, more work must be conducted in tropical regions. Studies in temperate areas (Biesmeijer et al. 2006; McFrederick \& LeBuhn 2006; Ahrné et al. 2009; Frankie et al. 2009; Kearns \& Oliveras 2009; Matteson \& Langellotto 2009, 2011; Bates et al. 2011; Hennig \& Ghazoul 2011; Hanley et al. 2014; Lowenstein et al. 2014; Pardee \& Philpott 2014; Baldock et al. 2015; Hülsmann et al. 2015; Banaszak-Cibicka et al. 2016; Plascencia \& Philpott 2017) have far outpaced studies in the tropics (Frankie et al. 2013), and we still know very little about the impacts of urbanization on tropical pollinator species. The two regions differ in a number of key attributes, such as the much greater biodiversity found in the tropics (Gaston 2000; Willig et al. 2003). Moreover, tropical urban pollinators may face different challenges than temperate urban pollinators, given that they remain active year-round (Bawa 1990). Previous studies have stressed the importance of 
86 pollination research in tropical cities (Vanbergen et al 2013; Harrison \& Winfree 2015),

87 particularly as most of the world's future population growth will occur in the tropics (Seto et al.

88 2012). Thus, the objective of this research was to examine the effect of key habitat factors (patch

89 size, floral abundance, plant richness, and location type) and landscape factors (percent

90 vegetation within $100,350,650,1,050$, and $1,550 \mathrm{~m}$ buffers) on the pollinator community within

91 a tropical megacity: Bangkok, Thailand.

92

93 METHODS

\section{Study Area}

Data were collected in Bangkok, Thailand from January through April 2017. Bangkok is by far the largest urban area in Thailand, with over 9.6 million residents inhabiting $2,100 \mathrm{~km}^{2}$ according to the most recent census in 2010 (World Bank). The landscape is dominated by manmade structures (business, residential, and transportation), and most of the vegetation found in Bangkok is intentionally cultivated and regularly managed. We selected 52 "green areas" (Table $\mathrm{S} 1$ ), defined as any location where vegetation comprises at least $50 \%$ of the ground area. We attempted to choose green areas evenly spaced throughout the city center (Fig. S1a), and these ranged in size from $600 \mathrm{~m}^{2}$ to $1,140,000 \mathrm{~m}^{2}$ (Table $\mathrm{S} 1$ ). We focused on the four most common types of green area: public parks, schools, temples, and commercial areas (Table S1).

\section{Pollinator Observations}

107 Within each green area, we conducted pollinator observations in $2 \times 2 \mathrm{~m}$ plots spaced throughout 108 the green area. Each plot was only observed once during the study period. Observations were 
109 conducted between 0600 and $1200 \mathrm{~h}$; in a separate study, we found that pollinator richness and

110 abundance did not differ significantly across time for 11 parks examined during both the

111 morning (0600-1200 h, $\mathrm{n}=119$ plots) and afternoon (1200-1800 h, $\mathrm{n}=102$ plots) (t-test;

112 richness: $t=0.24, P=0.8$; abundance: $t=0.12, P=0.9$ ). The number of plots per green area

113 (Table S1) was approximately proportional to the size of the green area (more plots were

114 conducted in larger green areas; Ahrné et al. 2009). In general, all plots within a green area were

115 sampled on the same day, except for green areas with more than 20 plots, which were conducted

116 over two consecutive days. To maximize the number of floral visitors encountered, we centered

117 plots in floral-rich areas, and sampled the flowers of as many plant species as possible. Each $2 \times 2$

$118 \mathrm{~m}$ plot was observed for 5 minutes, and if any visitors were observed during this period, we

119 continued observations for an additional 10 minutes. All animals visiting during the 15 -minute

120 observation period were recorded. We also noted whether or not visitors contacted floral

121 reproductive structures; visitors that contacted stigmas and anthers were considered potential

122 pollinators. Visitors that did not contact floral reproductive structures were not included in the

123 analyses. When possible, pollinators were identified to species, but some taxa (e.g., Tetragonula,

124 Lasioglossum) were difficult to identify to species in the field (and even in the lab), and were

125 therefore identified to genus or family. Unknown pollinators were either collected with a swing

126 net or photographed, and then identified with the help of entomologists (see acknowledgments).

127 Permission to work with animals was granted by MUSC-IACUC (Faculty of Science, Mahidol

128 University - Institute Animal Care and Use Committee) (license number MUSC60-038-388).

129 Pollinator richness was measured as the number of pollinator taxa per observation period,

130 and pollinator abundance was quantified as the number of pollinator individuals per observation 
131 period. The abundances of focal pollinator groups (stingless bees, honey bees, carpenter bees,

132 and butterflies) were also determined.

133

134 Habitat and Landscape Factors

135 We collected data on 4 habitat factors: patch size, location type, floral abundance, and plant

136 richness. Patch size and location type were measured at the level of the green area, while floral

137 abundance and plant richness were measured at the level of the plot. Patch size, or the total

138 expanse of land within each green area (not including the area covered by large bodies of water),

139 was measured using the "satellite" view in Google Maps. Each green area was also categorized

140 into one of four location types: public parks, schools, temples, and commercial areas. We

141 recorded both floral abundance (the number of flowers within each plot) and the floral

142 abundance of attractive plants (defined as plant species whose flowers were visited by at least

143 one pollinator). Because Bangkok has many cultivated plant species that are unattractive to

144 pollinators (Table S2), attractive floral abundance was considered more appropriate for

145 addressing our research objectives than total floral abundance. Indeed, compared to total floral

146 abundance, attractive floral abundance was found to be a better predictor of both pollinator

147 richness (Pearson correlation: $r=0.363$ versus $r=0.326$ ) and pollinator abundance (Pearson

148 correlation: $r=0.244$ versus $r=0.204)$. We therefore used the floral abundance of attractive

149 plants for all analyses (hereafter referred to as simply "floral abundance"). Plant richness was

150 quantified as the total number of plant species observed at all plots within a green area (a proxy

151 for total plant richness of the green area) divided by the number of plots conducted (to account

152 for the fact that more plots were observed in larger green areas). Plants were identified to species

153 or genus, not to the level of cultivar. 
For landscape factors, we calculated the percentage of vegetated area surrounding each

155

156

157

158

159

160

161

162

163

164

165

166

167

168

169

170

171

172

173

174

175

176

plot within 100, 350, 650, 1,050, and 1,550 m concentric circles (Fig. S1b). We used the

following secondary data sources: (a) Bangkok Metropolis map (1:4,000 scale) and (b) IKONOS

2017 satellite images at $1 \mathrm{~m}$ resolution. The satellite image was used to classify vegetated and

non-vegetated areas, and converted into digital format through supervised classification in

ArcGIS 10.4 software. We then used ArcCatalog to create a new geodatabase (containing spatial attributes) and data set (containing non-spatial attributes) for each spatial scale (100, 350, 650,

1,050 , and $1,550 \mathrm{~m}$ radiuses). The output was then used to calculate percent vegetation

(vegetated area divided by the sum of vegetated and non-vegetated areas) surrounding each plot at each spatial scale.

\section{Statistical Analysis}

All analyses were conducted in R 3.4.4 (R Core Team 2018). We used generalized linear mixed modeling (GLMM) to determine the effect of focal predictors on the following response variables: total pollinator richness, total pollinator abundance, and the abundance of each focal pollinator group (stingless bees, honey bees, carpenter bees, and butterflies). Green area was included as a random factor (as multiple plots were conducted per green area) and the Poisson distribution was used for all models (as all response variables were counts).

Model selection followed modified methods of Bates et al. (2011). For each spatial scale $(0,100,350,650,1,050$, and 1,550 $\mathrm{m}$ buffers $)$, nested likelihood ratio tests were used to determine the significance of each habitat factor of interest: patch size, location type, floral abundance, plant richness, the interaction between patch size and floral abundance, and the interaction between patch size and plant richness. We then selected the top model from each 
177 spatial scale and compared their AIC (Akaike information criterion) values to select the best

178 model overall (the model with the lowest AIC).

179 To visualize plant-pollinator interactions as a whole, and to analyze pollinator diet

180 breadth, we constructed and analyzed a pollination network using the "bipartite" package

181 (Dormann et al. 2008, 2009) in R 3.4.4. We pooled the interaction data of all 52 green areas and

182 used the function "plotweb" to build the network. We then used the function "specieslevel" to 183 examine diet generalization for each pollinator taxa with the metrics "degree" and "normalized

184 degree". Degree refers to the number of floral hosts per pollinator taxa. Normalized degree is 185 calculated as degree divided by the number of possible interacting partners, which accounts for differences in network size. Normalized degree is a quantitative measure of a pollinator species’ position in the network; species with high normalized degrees are central to network structure and promote network robustness (Sole \& Montoya 2001; Dunne et al. 2002). Moreover, high normalized degrees (near 1) indicate more generalized diets, while small degrees (near 0 ) indicate greater specialization.

\section{RESULTS}

Plant species

A total of 140 flowering plant taxa (46 families; Table S2) were observed from 469 plots in the 52 green areas. The most specious plant families observed in this study were Leguminosae (19 species), Apocynaceae (11 species), and Acanthaceae (9 species). Plots had an average of 221.2

$198 \pm 27.4$ flowers (range: 1 to 9,000 flowers per plot). The three most commonly observed plant 199 species were Ruellia simplex C.Wright (observed in 46 plots), Cassia fistula L. (32 plots), and 
200

201

202

203

204

205

206

207

208

209

210

211

212

213

214

215

216

217

218

219

220

221

\section{Habitat and landscape factors}

\section{Pollinator species} results.]

Caesalpinia pulcherrima (L.) Sw. (28 plots). The plant species that attracted the most pollinator individuals per flower (number of pollinators observed divided by number of flowers recorded in single species plots) were Gustavia gracillima Miers (22.2 pollinators per flower), Nymphaea nouchali Burm.f. (10.1 pollinators per flower), and Nelumbo nucifera Gaertn. (9.75 pollinators per flower). The plant species that attracted the greatest diversity of pollinator species were Citrus maxima (Burm.) Merr. (5 pollinator species), Justicia betonica L. (5 species), Buddleja paniculata Wall. (4.5 species), Peltophorum pterocarpum (DC.) K.Heyne (4.2 species), and Syzygium jambos (L.) Alston (4 species). [See Table S2 for more detailed results.]

Throughout this study, we observed a total of 18,793 pollinators comprising 40 taxa from 6 orders (Table S3). Hymenopterans (bees and wasps) were by far the most abundant, comprising 98.63\% of all pollinators observed. Lepidoptera (butterflies and moths; $1.16 \%$ ), Diptera (flies; 0.15\%), Coleoptera (beetles; 0.032\%), and Hemiptera (true bugs; 0.021\%) were also observed contacting floral reproductive structures, as was one sunbird (order Passeriformes, family Nectariniidae; $0.005 \%)$. The most common pollinators were stingless bees $(64.32 \%)$, of which only a single genus was observed (Tetragonula). Next most abundant were the three species of honey bees (Apis florea, 12.99\%; A. cerana, 12.48\%; A. dorsata, 6.11\%) and one species of carpenter bee (Xylocopa aestuans, 1.45\%). The abundances of the remaining pollinator taxa each accounted for less than 1\% of total pollinator observations. [See Table S3 for more detailed 
223 The effects of habitat and landscape factors were relatively similar for both pollinator richness

224 and abundance (Table 1; Fig. 1, 2). When combining the data of all pollinator taxa, both total

225 pollinator richness and total pollinator abundance were significantly influenced by patch size,

226 floral abundance, and their interaction (Table 1; Fig. 1, 2). However, total pollinator richness was

227 best described by the model including percent vegetation within a $650 \mathrm{~m}$ radius, while total

228 pollinator abundance was best described by the model including percent vegetation within a 350

229 m radius (Table 1).

230 When examining the abundance of focal taxonomic groups, the results were generally

231 consistent with those of total pollinator abundance (Table 1; Fig. S2a, S2c). The abundance of

232 honey bees and carpenter bees were each significantly influenced by the same three factors as

233 overall abundance: patch size, floral abundance, and their interaction (Table 1; Fig. S2a, S2c).

234 The abundance of butterflies was also affected by the same three factors, as well as location type

235 (Table 1; Fig. S2a-S2c). Finally, the abundance of stingless bees was influenced by floral

236 abundance and the interaction between patch size and floral abundance (but not patch size itself;

237 Table 1; Fig. S2a, S2c). The results for percent vegetation were more variable; stingless bees

238 were most influenced at the $100 \mathrm{~m}$ scale, butterflies and carpenter bees at the $350 \mathrm{~m}$ scale, and

239 honey bees at the $650 \mathrm{~m}$ scale (Table 1).

240 Significant habitat and landscape factors nearly always resulted in the same pattern for

241 the different taxonomic groups (Fig. 1, 2, S2a, S2c). Larger patch size and greater floral

242 abundance, when significant, increased pollinator richness and abundance (Fig. 1, S2a). On the

243 other hand, greater percent vegetation generally reduced pollinator richness and abundance,

244 although its effect on the abundance of carpenter bees and butterflies was minimal (Fig. 1, S2a).

245 Regarding significant interactions between patch size and floral abundance, greater floral 
246 abundance increased both pollinator richness and abundance in the larger patches, but did not

247 increase these metrics in the smallest patches (Fig. 2, S2c).

248

249 Pollination network

250 Our pollination network (Fig. 3, S3) revealed that Tetragonula, the three Apis species,

251 and $X$. aestuans were not only the most common pollinators, but also visited a high diversity of

252 plant species. Examining the network metrics confirmed that these taxa had the most generalized

253 diets: Tetragonula visited 93 plant species, followed by A. cerana (42 species), A. florea (33

254 species), X. aestuans (19 species), and A. dorsata (17 species). [See Table S4 for more detailed

255 results.]

256

257

DISCUSSION

258

259

Pollinator abundance and richness

260

Pollinator abundance was surprisingly high, given the extent of urbanization in Bangkok. We

261

observed an average of $40.1 \pm 2.8$ (mean \pm SE, $n=469$ plots) pollinators per $2 \times 2 \mathrm{~m}$ plot per 15 -

262

minute observation period (range: 0-422 pollinators). In comparison, Hennig and Ghazoul (2011)

263

reported an average of 32 visits per $2 \times 2 \mathrm{~m}$ plot (observed for 40 minutes each). The high

264

pollinator abundances observed in this study were due in large part to the Tetragonula stingless

265

bees; it was not uncommon to observe between 100-300 stingless bees at plots with high

266

abundances of attractive flowers. The three Apis species were occasionally observed in high

267

numbers (up to around 100 individuals of a single Apis species), but it was extremely uncommon

268 for any of the other taxa to exceed 10 individuals per observation period. 

reported in previous urban studies. Hennig and Ghazoul (2011) observed 148 insect species in Zürich, Switzerland, while Baldock et al. (2015) noted 147 visitor taxa at 12 urban sites

272 throughout the UK. Moreover, reports of urban bee species richness alone have ranged between

273 37-64 species (Kearns \& Oliveras 2009; Bates et al. 2011; Matteson et al. 2008; Frankie et al.

274 2013; Lowenstein et al. 2014). There are likely two factors contributing to the low richness

275 observed in this study. Firstly, many common taxa could only be classified to genus or family in 276 the field (e.g., Tetragonula, Lassioglossum, Lycaenidae; Table S3). More specific identification 277 would undoubtedly raise our measures of species richness. Secondly, Bangkok's lower richness is not surprising given the extent of urbanization in the city. Most of the studies mentioned above

279 were conducted in much smaller cities (less than three million residents), with the exception of 280 the study by Matteson et al. (2008) that was conducted in New York City. Thus, we predict that 281 only pollinator species unfazed by a highly anthropogenic environment can persist in Bangkok. The most common species observed in Bangkok do appear to be species with generalist diets and adaptable nesting behaviors. The reason we suggest a generalist diet is important is because the floral environment is almost always highly managed and frequently altered. Yet regularly switching blooming plant species may actually benefit generalist, urban pollinators by providing them with a continuous supply of floral resources. This constant floral supply may be particularly beneficial in tropical areas such as Bangkok, where pollinator species forage yearround, unlike temperate pollinators which are only active during the warmer months (Bawa 1990). The pollination network constructed from our data reveals that the most common insect species do indeed have broad diets. Moreover, all of the common bee species observed in our 
292 colony that had made their home in a small, plastic electrical box. Previous work has also

293 reported stingless bees (Heard 1999), A. cerana (Inoue \& Salmah 1990), A. dorsata (Deodikar et

294 al. 1977; Nagaraja \& Yathisha 2015), A. florea (Wongsiri et al. 1997), and Xylocopa bees

295 (Watmough 1973) utilizing man-made structures for nesting.

296

297 Effects of patch size, floral abundance, and plant richness

298 The two main effects that consistently had a positive influence on the pollinator indices examined were patch size and floral abundance. These findings are generally consistent with the

300

301

302

303

304

305

306

307

308

309

310

311

312

313 results of previous studies. Floral abundance appears to be the most universally positive factor, significantly influencing bees (McFrederick \& LeBuhn 2006; Ahrné et al. 2009; Matteson \& Langellotto 2010; Bates et al. 2011; Hennig \& Ghazoul 2011; Pardee \& Philpott 2014), hoverflies (Bates et al. 2011; Hennig \& Ghazoul 2011), and butterflies (Hardy \& Dennis 1999;

Matteson \& Langellotto 2010). Patch size is less consistent; some studies have found that measures of pollinator richness or abundance increase with larger patches (Hennig \& Ghazoul 2011; Pardee \& Philpott 2014), while others have reported that patch size has no effect on pollinators (McFrederick \& LeBuhn 2006; Ahrné et al. 2009). Findings for plant richness have also been mixed, with some studies reporting minimal effects (Banaszak-Cibicka et al. 2016; Plascencia \& Philpott 2017), as in our study, and others reporting a significant positive effect (Kearns \& Oliveras 2009; Bates et al. 2011; Hennig \& Ghazoul 2011; Hülsmann et al. 2015). In Bangkok, it appears that floral abundance is more important that plant richness, which may be explained by the apparent flexible diets of most observed pollinator species. Moreover, the importance of floral abundance and patch size likely results from the fact that the former is a 
314 direct measure of food availability and the latter is possibly correlated with nest site availability,

315 both of which are important to pollinators (Baldock et al. 2015).

317 Interaction between patch size and floral abundance

318 Interestingly, our results also revealed a consistent significant interaction between patch size and

319 floral abundance. The interaction revealed, for the larger green areas, a strong positive

320 correlation between floral abundance and all pollinator indices. However, for the smallest green

321 areas, increasing floral abundance did not greatly increase pollinator richness or abundance. We

322 suggest three potential explanations for these patterns. Firstly, since pollinator richness increases

323 with floral abundance in the large areas but not the small areas, we suggest that small green areas

324 lack the diversity of nesting sites needed to recruit greater pollinator richness. Secondly, since

325 the same pattern occurs for pollinator abundance, we suggest that pollinators do not become

326 more abundant with increasing floral abundance in the smallest green areas due to limited

327 nesting availability in these areas. Finally, it is possible that some pollinator species prefer larger

328 foraging grounds than what is offered by small green areas (Pauw 2007). Previous studies have

329 not looked for an interaction between patch size and floral abundance, and further studies are

330 required to determine if such patterns are common in other urban areas.

332 Effect of percent vegetation

333 The spatial scale that best fit our pollinator richness and abundance data differed by taxa, which

334 likely reflects their different foraging ranges. Tetragonula stingless bees (best described by

335 percent vegetation within $100 \mathrm{~m}$ ) are relatively small pollinators with correspondingly small

336 foraging distances (120-850 m; van Nieuwstadt et al. 1996; Smith et al. 2017). Kuhn-Neto et al. 
337 (2009), however, did report that the stingless bee Melipona mandacaia could forage up to 2.1

$338 \mathrm{~km}$. Xylocopa carpenter bees and butterflies (best described at the $350 \mathrm{~m}$ scale) have been

339 reported to have longer foraging distances. For example, Pasquet et al. (2008) found that

340 Xylocopa flavorufa could forage between 50 to $6,040 \mathrm{~m}$ (median $=720 \mathrm{~m}$ ), Cant et al. (2005)

341 observed that the style of butterfly flight characterized by foraging activity spanned 1,210.9 \pm

$342455.4 \mathrm{~m}$, and Ovaskainen et al. (2008) reported that Melitaea cinxia butterflies flew between 9 to

$3435,490 \mathrm{~m}($ median $=505 \mathrm{~m})$. Apis honey bees (best described at the $650 \mathrm{~m}$ scale) have even longer

344 maximum foraging distances: at least $1 \mathrm{~km}$, and potentially up to $12 \mathrm{~km}$ (Dyer \& Seeley 1991).

345 While the spatial scales that best fit our models are smaller than previously reported foraging

346 distances, it is consistent with prior work which has suggested that pollinators in urban areas may

347 have smaller foraging ranges than those in natural habitats (López-Uribe et al. 2008).

348 The negative correlation between percent vegetation and our various pollinator indices

349 was contrary to our initial hypothesis. Indeed, previous studies have generally found that greater

350 vegetation in the surrounding landscape increases measures of pollinators or pollination success

351 (Bates et al. 2011; Sritongchuay et al. 2016). However, we suggest that the vegetation in

352 Bangkok is inherently different than the natural, uncultivated vegetation encountered in previous

353 studies. First of all, much of the vegetation in Bangkok consists of hedges and lawns, which

354 often do not offer floral resources. Moreover, many species considered attractive to humans and

355 planted in abundance throughout the city were observed to be highly unattractive to insects (e.g.,

356 Bougainville, Plumeria, Hibiscus; Table S2). Finally, much of Bangkok's vegetation is highly

357 managed (hedges and trees are trimmed and lawns are mown), which may deter insect

358 pollinators from utilizing them as either nesting or foraging sites. Similar to our results,

359 Banaszak-Cibicka et al. (2016) also reported a negative correlation between urban bee density 
360 and shrub cover, and Matteson \& Langellotto (2010) found no effect of landscape level

361 vegetation on bee richness. While urban vegetation certainly provides other benefits (Smith et al.

362 2006; Robinson \& Lundholm 2012), it does not necessarily promote pollinator richness or

363 abundance.

364

365 Effect of location type

366 Most pollinator taxa did not appear to differentiate between the various location types (public

367 parks, schools, temples, and commercial areas). The one exception was the butterflies, which

368 were more common in public parks than the other three location types. Butterflies were the most

369 skittish insect pollinators that we observed, so human density or activity may have reduced

370 butterfly abundance at schools, temples, and commercial areas (although this is just speculation

371 as we did not quantify human density or activity). However, all other pollinator groups were

372 unaffected by location type. There are two key factors that likely contribute to these findings.

373 The first is that Bangkok's schools, temples, and other commercial areas typically have higher

374 floral abundances than the surrounding urban landscape, and share similar characteristics with

375 public parks. Notably, in our study, there were no significant differences among the four location

376 types for either floral abundance (ANOVA, $F=1.39, P=0.26$ ) or patch size (ANOVA, $F=2.1$,

$377 P=0.11)$. The second key factor is that the pollinator taxa observed in Bangkok are relatively

378 generalist species, with flexible foraging and nesting requirements (as covered at the beginning

379 of the Discussion). Our results indicate that school campuses, temples, and even commercial

380 areas can be just as effective as public parks in promoting urban pollinator richness and

381 abundance. 


\section{Native versus exotic plant species}

384 While not the focus of this study, we did also compare pollinator preferences for native $(n=43$

385 species) versus exotic $(n=65$ species $)$ plants. Overall pollinator richness and abundance, as well

386 as abundance of each of the focal taxa, were not significantly different between native and exotic

387 plant species (Table S5). Previous studies have reported mixed findings. For example, Chrobock

388 et al. (2013) found that pollinator visitation rates to native plant species were higher than those to

389 exotic species, while Matteson and Langellotto (2011) reported that bumble bees and Apis

390 mellifera visited native and exotic species equally, yet megachilid bees and butterflies actually

391 favored exotic plant species. Moreover, Hanley et al. (2014) found that bumblebee species with

392 specialized diets preferred native species and bumblebee species with generalized diets preferred

393 exotic species, and Pardee and Philpott (2014) reported that native bee species preferred native

394 gardens over non-native gardens. While all of the pollinator species in our study (that we had

395 information for) were native (Table S3), their lack of preference between native and exotic plant

396 species may be explained in part by their generalized diets. Moreover, given their prevalence in

397 Bangkok, exotic plants may be important in sustaining the city’s pollinator populations, as has

398 been suggested for other urban areas (Shapiro 2002; Matteson \& Langellotto 2011; Harrison \&

399 Winfree 2015).

400

401 Conclusions

402 The results of this study provide important information about pollinator communities in a highly-

403 populated, tropical city, which are vastly understudied compared to their temperate counterparts.

404 The habitat factors exerting the largest influence on pollinator richness and abundance were

405 patch size and floral abundance, yet the significant interaction between these two factors 
406 revealed that the smallest green areas (ca. $\left.<10,000 \mathrm{~m}^{2}\right)$ retained low pollinator richness and

407 abundance, even when floral abundance was very high. Such findings highlight the importance

408 of retaining large green areas in cities. Yet this work also reveals that the meaning of the term

409 "green area" is not necessarily restricted to traditional public parks, as other patch types with

410 high floral abundance can support urban pollinators. We suggest additional work examining

411 pollinator communities in large cities, particularly in the tropics, where most of the world's

412 population growth is expected to occur.

413

414 ACKNOWLEDGMENTS

415

416 We thank Dr. Natapot Warrit and Dr. Tom Stewart for helping identify insect specimens and

417 photos, and Dr. Prapeut Kerdsueb for helping with GIS analyses. We also thank Dr. Gail

418 Langellotto and one anonymous reviewer for comments on an earlier draft. Permission to work

419 with animals was granted by MUSC-IACUC (Faculty of Science, Mahidol University - Institute

420 Animal Care and Use Committee) (license number MUSC60-038-388).

421 


\section{REFERENCES}

423

424 Ahrné, K., J. Bengtsson, and T. ElmQvist. 2009. Bumble Bees (Bombus spp) along a Gradient 425 of Increasing Urbanization. PLoS ONE 4: e5574.

426

427

428

BALdock, K.C.R., M.A. GoddARD, D.M. Hicks, W.E. Kunin, N. MitschunAs, L.M.

Osgathorpe, S.G. Potts, K.M. Robertson, A.V. Scott, G.N. Stone, I.P. VAughan, and J. Memmotт. 2015. Where is the UK's pollinator biodiversity? The importance of urban areas for flower-visiting insects. Proceedings of the Royal Society B 282: 20142849.

BANASZAK-CIBICKA, W., H. RATYŃSKA, and Ł. DYLEWSKI. 2016. Features of urban green space favourable for large and diverse bee populations (Hymenoptera: Apoidea: Apiformes). Urban Forestry \& Urban Greening, 20: 448-452.

Bates, A.J., J.P. SAdler, A.J. Fairbrass, S.J. FAlK, J.D. Hale, and T.J. Matthews. 2011. Changing Bee and Hoverfly Pollinator Assemblages along an Urban-Rural Gradient. Plos One 6: e23459.

BAWA, K.S. 1990. Plant-pollinator interactions in tropical rain forests. Annual Review of Ecology and Systematics 21: 399-422.

Bhattacharya, M., R.B. Primack, and J. GerWEIN. 2003. Are roads and railroads barriers to bumblebee movement in a temperate suburban conservation area? Biological Conservation 109: $37-45$.

Biesmeijer, J.C., S.P.M. Roberts, M. Reemer, R. Ohlemüller, M. Edwards, T. Peeters, A.P. Schaffers, S.G. Potts, R. Kleukers, C.D. Thomas, J. Settele, and W.E. Kunin. 2006. Parallel Declines in Pollinators and Insect-Pollinated Plants in Britain and the Netherlands. 
CAnt, E.T., A.D. SMith, D.R. ReynOldS, and J.L. Osborne. 2005. Tracking butterfly flight paths across the landscape with harmonic radar. Proceedings of the Royal Society B 272: $785-790$.

CARIVEAU, D.P., and R. WinfREE. 2015. Causes of variation in wild bee responses to anthropogenic drivers. Current Opinion in Insect Science 10: 104-109.

Chrobock, T., P. Winiger, M. Fischer, and M. VAn KLeUnen. 2013. The cobblers stick to their lasts: pollinators prefer native over alien plant species in a multi-species experiment. Biological Invasions 15: 2577-2588.

Deodikar, G.B., A.I. Ghatge, R.P. Phadke, D.B. Mahindre, K.K. Kshirsagar, K.S. Muvel, and C. V. THAKAR. 1977. Nesting behaviour of Indian honeybees III. Nesting behaviour of Apis dorsata Fab. Indian Bee Journal 39: 1-12.

DORMANN C.F., B. GRUBER, and J. FRUEND. 2008. Introducing the bipartite package: analyzing ecological networks. $R$ news, 8:8-11.

Dormann C.F., J. Fruend, N. BluethGen, and B. GRUBER. 2009. Indices, graphs and null models: analyzing bipartite ecological networks. Open Ecology Journal, 2:7-24.

DunNe, J.A., R.J. WiLliams, and N.D. MARTinEZ. 2002. Food-web structure and network theory: the role of connectance and size. Proceedings of the National Academy of Sciences, 99:12917-12922.

DyER, F.C., and T.D. SEELEY. 1991. Dance dialects and foraging range in three Asian honey bee species. Behavioral Ecology and Sociobiology 28: 227-233.

FERREIRA, P.A., D. Boscolo, and B.F. ViAnA. 2013. What do we know about the effects of landscape changes on plant-pollinator interaction networks? Ecological Indicators 31: 3540. 
468 Frankie, G.W., R.W. Thorp, J. Hernandez, M. Rizzardi, J.C. PaWelek, S.L. Witt, R.

469 COVILLE, and V.A. WoJCIK. 2009. Native bees are a rich natural resource in urban

$470 \quad$ California gardens. California Agriculture 63: 113-120.

471 Frankie, G.W., S.B. Vinson, M.A. Rizzardi, T.L. Griswold, R.E. Coville, M.H. Grayum,

472

473

474

475

476

477

478

479

480

481

482

483

484

485

486

487

488

489

490

L.E.S. MartineZ, J. FolTZ-SwEAT, and J.C. PAWELEK. 2013. Relationships of Bees to Host

Ornamental and Weedy Flower Urban Northwest Guanacaste Province, Costa Rica. Journal of the Kansas Entomological Society 86: 325-351.

GASTON, K.J. 2000. Global patterns in biodiversity. Nature 405: 220-227.

Grimm, N.B., S.H. FAeth, N.E. Golubiewski, C.L. Redman, J. Wu, X. BAi, and J.M. BriggS. 2008. Global Change and the Ecology of Cities. Science 319: 756-760.

Hall, D.M., G.R. Camilo, R.K. Tonietto, J. Ollerton, K. Ahrné, M. Arduser, J.S. Ascher, K.C.R. Baldock, R. Fowler, G. Frankie, D. Goulson, B. Gunnarsson, M.E. Hanley, J.I. Jackson, G. Langellotto, D. Lowenstein, E.S. Minor, S.M. Philpott, S.G. Potts, M.H. Sirohi, E.M. SpevaK, G.N. Stone, and C.G. Threlfall. 2016. The city as a refuge for insect pollinators. Conservation Biology 31: 24-29.

Hanley, M.E., A.J. AwBI, and M. FranCO. 2014. Going native? Flower use by bumblebees in English urban gardens. Annals of Botany 113: 799-806.

HARDY, P.B., and R.L.H. DENNIS. 1999. The impact of urban development on butterflies within a city region. Biodiversity and Conservation 8: 1261-1279.

HARRISON, T., and R. WiNFREE. 2015. Urban drivers of plant-pollinator interactions. Functional Ecology 29: 879-888.

HEARD, T.A. 1999. The role of stingless bees in crop pollination. Annual Review of Entomology183-206. 
491 HenNig, E.I., and J. GHaZOUL. 2011. Pollinating animals in the urban environment. Urban $492 \quad$ Ecosystems 15: 149-166.

493

494

495

496

497

498

499

500

501

502

503

504

505

506

507

508

509

510

511

512

Hülsmann M., H. Von Wehrden, A.M. Klein, and S.D. LeOnhardT. 2015. Plant diversity and composition compensate for negative effects of urbanization on foraging bumble bees. Apidologie, 46: 760-770.

InOUE, T., AdRI, and S. SAlmaH. 1990. Nest site selection and reproductive ecology of the Asian honey bee, Apis cerana indica, in central Sumatra. In S. F. Sakagami, R. I. Ohgushi, and D. W. Roubik [eds.], Natural history of social wasps and bees in equatorial Sumatra, 219-232. Hokkaido University Press, Sapporo, Japan.

KEARNS, C.A., and D.M. OLIVERAS. 2009. Environmental factors affecting bee diversity in urban and remote grassland plots in Boulder, Colorado. Journal of Insect Conservation 13: 655665.

Klein, A.-M., B.E. Vaissière, J.H. CAne, I. Steffan-Dewenter, S.A. Cunningham, C. KREMEN, and T. TSCHARNTKE. 2007. Importance of pollinators in changing landscapes for world crops. Proceedings of the Royal Society B 274: 303-313.

Kuhn-Neto, B., F.A.L. Contrera, M.S. CAStro, and J.C. NiEH. 2009. Long distance foraging and recruitment by a stingless bee, Melipona mandacaia. Apidologie 40: 472-480.

LÓPEZ-UriBe, M.M., C.A. OI, and M.A. Del LAMA. 2008. Nectar-foraging behavior of Euglossine bees (Hymenoptera: Apidae) in urban areas. Apidologie 39: 410-418.

Lowenstein, D.M., K.C. Matteson, I. XiaO, A.M. Silva, and E.S. MinOR. 2014. Humans, bees, and pollination services in the city: The case of Chicago, IL (USA). Biodiversity and Conservation 23: 2857-2874. 
513 Matteson, K.C., J.S. Ascher, and G.A. Langellotto. 2008. Bee Richness and Abundance in

$514 \quad$ New York City Urban Gardens. Annals of the Entomological Society of America 101: 140$515 \quad 150$.

516 Matteson, K.C., and G.A. Langellotto. 2009. Bumble bee abundance in New York City 517 community gardens: implications for urban agriculture. Cities and the Environment 2: 1-12.

518 Matteson, K.C., and G.A. LAngellotto. 2010. Determinates of inner city butterfly and bee $519 \quad$ species richness. Urban Ecosystems 13: 333-347.

520 Matteson, K.C., and G.A. Langellotto. 2011. Small scale additions of native plants fail to 521 increase beneficial insect richness in urban gardens. Insect Conservation and Diversity 4: $522 \quad 89-98$.

523 MCFrederick, Q.S., and G. LEBUHN. 2006. Are urban parks refuges for bumble bees Bombus 524 spp. (Hymenoptera: Apidae)? Biological Conservation 129: 372-382.

525 Nagaraja, N., and V. YAthisha. 2015. Nest orientation of Asian giant honeybee, Apis dorsata 526 in plains of Karnataka, India. Journal of Entomological Research 39: 197-201.

527 Ollerton, J., R. Winfree, and S. TARRANT. 2011. How many flowering plants are pollinated by $528 \quad$ animals? Oikos 120: 321-326.

529 Ovaskainen, O., A.D. Smith, J.L. Osborne, D.R. Reynolds, N.L. CArreck, A.P. Martin, K. 530 NiITEPOLD, and I. HANSKI. 2008. Tracking butterfly movements with harmonic radar 531 reveals an effect of population age on movement distance. Proceedings of the National $532 \quad$ Academy of Sciences 105: 19090-19095.

533 PARDEE, G.L., and S.M. PhiLpott. 2014. Native plants are the bee's knees: local and landscape 534 predictors of bee richness and abundance in backyard gardens. Urban Ecosystems 17: 641$535 \quad 659$. 
536 Pasquet, R.S., A. Peltier, M.B. Hufford, E. Oudin, J. Saulnier, L. Paul, J.T. Knudsen, H.R. 537 HERREN, and P. GEPTS. 2008. Long-distance pollen flow assessment through evaluation of 538 pollinator foraging range suggests transgene escape distances. Proceedings of the National $539 \quad$ Academy of Sciences 105: 13456-13461.

540 PAuw, A. 2007. Collapse of a Pollination Web in Small Conservation Areas. Ecology 88: 1759$541 \quad 1769$.

542 Plascencia M., S.M. Philpott. 2017. Floral abundance, richness, and spatial distribution drive 543 urban garden bee communities. Bulletin of Entomological Research, 107: 658-667.

544 Potts, S.G., J.C. Biesmeijer, C. Kremen, P. Neumann, O. Schweiger, and W.E. Kunin. 2010. 545 Global pollinator declines: trends, impacts and drivers. Trends in Ecology \& Evolution 25: $546 \quad 345-353$.

547 R CORE TEAM. 2018. R: A language and environment for statistical computing. R Foundation for 548 Statistical Computing, Vienna, Austria. Available at https://www.R-project.org/.

549 RoBInSON, S.L., and J.T. LunDHOLM. 2012. Ecosystem services provided by urban spontaneous $550 \quad$ vegetation. Urban Ecosystems 15: 545-557.

551 Seto, K.C., B. GÜNERALP, and L.R. HutYRA. 2012. Global forecasts of urban expansion to 2030 552 and direct impacts on biodiversity and carbon pools. Proceedings of the National Academy $553 \quad$ of Sciences 109: 16083-16088.

554 SHAPIRO, A.M. 2002. The Californian urban butterfly fauna is dependent on alien plants.

$555 \quad$ Diversity and Distributions 8: 31-40.

556 Smith, J.P., T.A. HeArD, M. BeEkMAN, and R. GloAg. 2017. Flight range of the Australian 557 stingless bee Tetragonula carbonaria (Hymenoptera: Apidae). Austral Entomology 56: 50$558 \quad 53$. 
559 Smith, R.M., K. Thompson, J.G. Hodgson, P.H. WArren, and K.J. Gaston. 2006. Urban

560 domestic gardens (IX): composition and richness of the vascular plant flora, and

561 implications for native biodiversity. Biological Conservation 129: 312-322.

562 Sole, R.V., and J.M. Montoya. 2001. Complexity and fragility in ecological networks.

563 Proceedings of the Royal Society B, 268:2039-2045.

564 Sritongchuay, T., C. KREMEN, and S. BUMRUNGSRI. 2016. Effects of forest and cave proximity

565 on fruit set of tree crops in tropical orchards in Southern Thailand. Journal of Tropical

566 Ecology 32: 269-279.

567 VAN NiEUWSTADT, M., and C.R. IRAHETA. 1996. Relation between size and foraging range in

568 stingless bees (Apidae, Meliponinae). Apidologie 27: 219-228.

569 VANBERGEN, A.J. 2013. Threats to an ecosystem service: pressures on pollinators. Frontiers in $570 \quad$ Ecology and the Environment 11:251-259.

571 Watmough, R.H. 1973. Biology and behaviour of carpenter bees in southern Africa. Journal of 572 the Entomological Society of southern Africa 37: 261-281.

573 Willig, M.R., D.M. Kaufman, and R.D. Stevens. 2003. Latitudinal gradients of biodiversity:

574 pattern, process, scale, and synthesis. Annual Review of Ecology, Evolution, and

$575 \quad$ Systematics 34: 273-309.

576 Wongsiri, S., C. LEKPrAyoOn, R. Thapa, K. ThiRAKuPt, T.E. Rinderer, H.A. Sylvester, 577 B.P. OldRoyd, and U. BoONCHAM. 1997. Comparative biology of Apis andreniformis and 578 Apis florea in Thailand. Bee World 78: 23-35.

579 WORLD BANK. 2015. Urbanization in Thailand is dominated by the Bangkok urban area.

580 Available at: http:/www.worldbank.org/en/news/feature/2015/01/26/urbanization-in-

581 thailand-is-dominated-by-the-bangkok-urban-area (accessed 20 May 2018). 


\section{Table $\mathbf{1}$ (on next page)}

Results of the generalized linear mixed models (GLMM) for our six response variables.

The response variables were: total pollinator richness, total pollinator abundance, and the abundances of 4 focal taxa (Tetragonula stingless bees, Apis honey bees, Xylocopa carpenter bees, and butterflies). The significance of the six habitat predictors ( 4 main factors and 2 interactions) were tested using nested likelihood ratio tests. The landscape scale (surrounding percent vegetation at $0,100,350,650,1,050$, and 1,550 m buffers) that best fit each model was determined from AIC values. The significant factors that were included in the final models are highlighted in yellow. 
1 Table 1. Results of the generalized linear mixed models (GLMM) for our six response variables.

2 The response variables were: total pollinator richness, total pollinator abundance, and the

3 abundances of 4 focal taxa (Tetragonula stingless bees, Apis honey bees, Xylocopa carpenter

4 bees, and butterflies). The significance of the six habitat predictors ( 4 main factors and 2

5 interactions) were tested using nested likelihood ratio tests. The landscape scale (surrounding

6 percent vegetation at $0,100,350,650,1,050$, and $1,550 \mathrm{~m}$ buffers) that best fit each model was

7 determined from AIC values. The significant factors that were included in the final models are

8 highlighted in yellow.

9

\begin{tabular}{|c|c|c|c|c|c|c|c|}
\hline & & $\begin{array}{c}\text { Total } \\
\text { Pollinator } \\
\text { Richness }\end{array}$ & $\begin{array}{c}\text { Total } \\
\text { Pollinator } \\
\text { Abundance }\end{array}$ & $\begin{array}{c}\text { Stingless } \\
\text { Bee } \\
\text { Abundance }\end{array}$ & $\begin{array}{l}\text { Honey Bee } \\
\text { Abundance }\end{array}$ & $\begin{array}{c}\text { Carpenter } \\
\text { Bee } \\
\text { Abundance }\end{array}$ & $\begin{array}{c}\text { Butterfly } \\
\text { Abundance }\end{array}$ \\
\hline \multirow{6}{*}{$\frac{\mathscr{T}}{\mathscr{T}}$} & $\begin{array}{c}\text { Patch Size } \\
\text { x Floral } \\
\text { Abundance } \\
\end{array}$ & $\begin{array}{c}\chi_{1}^{2}=5.48 \\
\mathbf{P}=\mathbf{0 . 0 1 9}\end{array}$ & $\begin{array}{l}\chi_{1}^{2}=1,546 \\
\mathbf{P}<\mathbf{0 . 0 0 1}\end{array}$ & $\begin{array}{c}\chi_{1}^{2}=956 \\
\mathbf{P}<\mathbf{0 . 0 0 1}\end{array}$ & $\begin{array}{c}\chi_{1}^{2}=530 \\
\mathbf{P}<\mathbf{0 . 0 0 1}\end{array}$ & $\begin{array}{l}\chi_{1}^{2}=81.3 \\
\mathbf{P}<\mathbf{0 . 0 0 1}\end{array}$ & $\begin{array}{l}\chi_{1}^{2}=83.0 \\
\mathbf{P}<\mathbf{0 . 0 0 1}\end{array}$ \\
\hline & $\begin{array}{c}\text { Patch Size } \\
\text { x Plant } \\
\text { Richness } \\
\end{array}$ & $\begin{array}{c}\chi_{1}^{2}=0.98 \\
\mathrm{P}=0.322\end{array}$ & $\begin{array}{l}\chi_{1}^{2}=1.20 \\
\mathrm{P}=0.273\end{array}$ & $\begin{array}{l}\chi_{1}^{2}=0.60 \\
\mathrm{P}=0.440\end{array}$ & $\begin{array}{c}\chi_{1}^{2}=0.19 \\
\mathrm{P}=0.660\end{array}$ & $\begin{array}{l}\chi_{1}^{2}=0.29 \\
\mathrm{P}=0.589\end{array}$ & $\begin{array}{c}\chi_{1}^{2}=0.98 \\
\mathrm{P}=0.322\end{array}$ \\
\hline & Patch Size & $\begin{array}{c}\chi_{1}^{2}=10.1 \\
\mathbf{P}=\mathbf{0 . 0 0 2}\end{array}$ & $\begin{array}{c}\chi_{1}^{2}=6.04 \\
\mathbf{P}=\mathbf{0 . 0 1 4}\end{array}$ & $\begin{array}{c}\chi_{1}^{2}=2.74 \\
\mathrm{P}=0.098\end{array}$ & $\begin{array}{c}\chi_{1}^{2}=7.82 \\
\mathbf{P}=\mathbf{0 . 0 0 5}\end{array}$ & $\begin{array}{c}\chi_{1}^{2}=4.38 \\
\mathbf{P}=\mathbf{0 . 0 3 6}\end{array}$ & $\begin{array}{c}\chi_{1}^{2}=10.9 \\
\mathbf{P}<\mathbf{0 . 0 0 1}\end{array}$ \\
\hline & $\begin{array}{c}\text { Floral } \\
\text { Abundance }\end{array}$ & $\begin{array}{c}\chi_{1}^{2}=23.6 \\
\mathbf{P}<\mathbf{0 . 0 0 1}\end{array}$ & $\begin{array}{l}\chi_{1}^{2}=2,576 \\
\mathbf{P}<\mathbf{0 . 0 0 1}\end{array}$ & $\begin{array}{l}\chi_{1}^{2}=1,506 \\
\mathbf{P}<\mathbf{0 . 0 0 1}\end{array}$ & $\begin{array}{c}\chi_{1}^{2}=641 \\
\mathbf{P}<\mathbf{0 . 0 0 1}\end{array}$ & $\begin{array}{c}\chi_{1}^{2}=122 \\
\mathbf{P}<\mathbf{0 . 0 0 1}\end{array}$ & $\begin{array}{c}\chi_{1}^{2}=139 \\
\mathbf{P}<\mathbf{0 . 0 0 1}\end{array}$ \\
\hline & $\begin{array}{c}\text { Plant } \\
\text { Richness }\end{array}$ & $\begin{array}{l}\chi_{1}^{2}=0.29 \\
\mathrm{P}=0.593\end{array}$ & $\begin{array}{l}\chi_{1}^{2}=0.12 \\
\mathrm{P}=0.729\end{array}$ & $\begin{array}{l}\chi_{1}^{2}=0.14 \\
\mathrm{P}=0.711\end{array}$ & $\begin{array}{c}\chi_{1}^{2}=0.21 \\
\mathrm{P}=0.649\end{array}$ & $\begin{array}{l}\chi_{1}^{2}=2.87 \\
\mathrm{P}=0.090\end{array}$ & $\begin{array}{c}\chi_{1}^{2}=3.41 \\
\mathrm{P}=0.065\end{array}$ \\
\hline & $\begin{array}{c}\text { Location } \\
\text { Type }\end{array}$ & $\begin{array}{c}\chi_{3}^{2}=3.29 \\
\mathrm{P}=0.349\end{array}$ & $\begin{array}{c}\chi_{3}^{2}=1.44 \\
\mathrm{P}=0.697\end{array}$ & $\begin{array}{c}\chi_{1}^{2}=0.24 \\
\mathrm{P}=0.971\end{array}$ & $\begin{array}{l}\chi_{1}^{2}=1.85 \\
\mathrm{P}=0.603\end{array}$ & $\begin{array}{c}\chi_{1}^{2}=1.45 \\
\mathrm{P}=0.695\end{array}$ & $\begin{array}{c}\chi_{1}^{2}=13.2 \\
\mathbf{P}=\mathbf{0 . 0 0 4}\end{array}$ \\
\hline \multirow{6}{*}{ 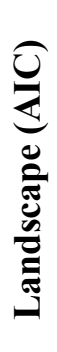 } & none & 1419 & 19029 & 12801 & 13822 & 1238 & 658 \\
\hline & $100 \mathrm{~m}$ & 1411 & 18686 & 12511 & 13713 & 1172 & 651 \\
\hline & $350 \mathrm{~m}$ & 1410 & 18511 & 12583 & 13448 & 1167 & 647 \\
\hline & $650 \mathrm{~m}$ & 1409 & 18561 & 12677 & 13315 & 1206 & 654 \\
\hline & $1,050 \mathrm{~m}$ & 1410 & 18807 & 12753 & 13504 & 1230 & 657 \\
\hline & $1,550 \mathrm{~m}$ & 1412 & 18944 & 12773 & 13690 & 1234 & 655 \\
\hline
\end{tabular}




\section{Figure 1 (on next page)}

Effects of significant habitat and landscape factors on total pollinator richness and abundance.

Two main factors (patch size and floral abundance) had significant positive effects on both richness ( $A-C$; number of species per 15-minute observation period) and abundance (D-F; number of individuals per hour), while one main factor (surrounding percent vegetation) had a significant negative effect. Surrounding percent vegetation at the $650 \mathrm{~m}$ radius best described pollinator richness, while the $350 \mathrm{~m}$ radius best described pollinator abundance. Each circle represents the mean of one green area (values averaged across all plots within the green area). 


\section{Figure 2 (on next page)}

The significant interaction between patch size and floral abundance.

Both (A) total pollinator richness (number of species per 15-minute observation period) and (B) total pollinator abundance (number of individuals per hour) were influenced by a significant interaction between patch size and floral abundance. Each circle represents the mean of one green area (values averaged across all plots within the green area). The different colors and line types represent the different patch size classes: light grey (solid line) = small green areas $\left(600-900 \mathrm{~m}^{2} ; \mathrm{n}=13\right)$, medium grey (dashed) $=$ medium size green areas $\left(901-30,000 \mathrm{~m}^{2} ; \mathrm{n}=13\right)$, dark grey (dot-dashed) $=$ large green areas $(30,001-81,000$ $\left.m^{2} ; n=13\right)$, black (dotted) = very large green areas $\left(81,001-1,140,000 m^{2} ; n=13\right)$. 


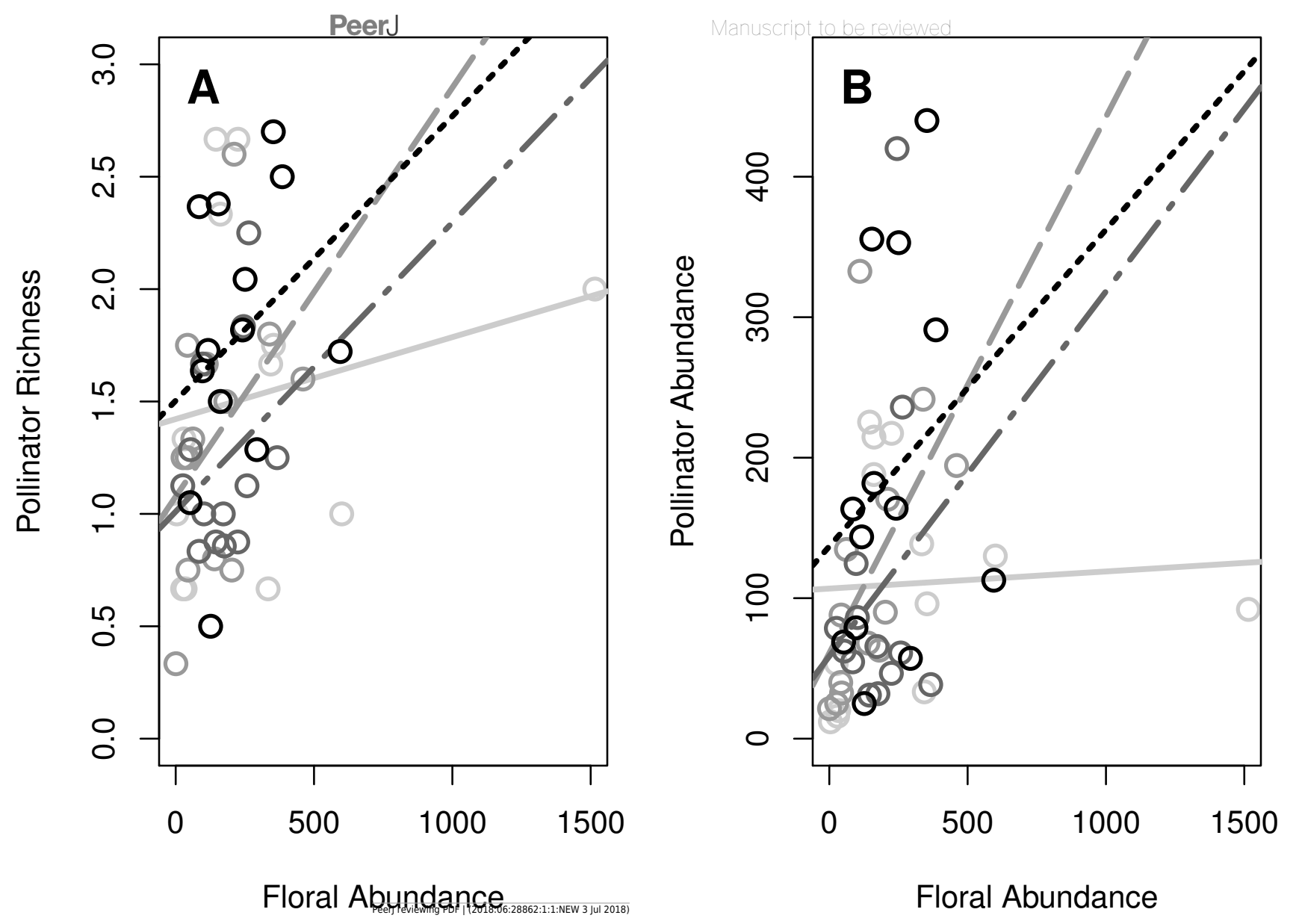




\section{Figure $\mathbf{3}$ (on next page)}

Pollination network of plants and pollinators in Bangkok, Thailand.

Pollinator taxa are listed along the top (yellow), and plant taxa are listed along the bottom (green). The network was constructed from pollinator abundance data at each plant species; a line connecting pollinator species $i$ to plant species $j$ represents an observed interaction between the two, and the width of the connecting line is proportional to the average number of $i$ pollinator individuals observed visiting plant species $j$. The five most abundant pollinator taxa were: $\mathrm{A} 1=$ Tetragonula, $\mathrm{A} 2=$ Apis florea, $\mathrm{A} 3=\mathrm{A}$. cerana, $\mathrm{A} 4=\mathrm{A}$. dorsata, and $\mathrm{A} 5=$ Xylocopa aestuans. [A higher resolution figure is available in Fig. S3, and all species names are listed in Table S4.] 
